\title{
Time-domain adaptive channel estimation for OFDM-based WLAN with multiple- antennas
}

\begin{abstract}
In this paper, an adaptive time-domain (TD) channel estimation scheme, based on recursive least squares (RLS) algorithm, is proposed for multiple-input multiple-output (MIMO) orthogonal frequency division multiplexing (OFDM) based wireless local area networks (WLANs). The estimator is then extended to perform decision-directed (DD) channel tracking during data transmission. The channel is assumed to be constant during one OFDM symbol but evolves in time according to the first-order Markov process. Different training rates at different Doppler frequencies were investigated. Simulation results show that the proposed estimation scheme has excellent performance measured in terms of the mean squares error (MSE) and the bit error rate (BER), provided that the forgetting factor of the RLS algorithm is optimally selected.
\end{abstract}

